\title{
Efecto sobre la salud de las desigualdades socioeconómicas en el adulto mayor. Resultados basales del estudio expectativa de vida saludable y discapacidad relacionada con la obesidad (Alexandros)
}

\author{
CECILIA ALBALA, HUGO SÁNCHEZ, LYDIA LERA a \\ BÁRBARA ANGEL ${ }^{\mathrm{b}}$, XIMENA CEA ${ }^{\mathrm{c}}$
}

Instituto de Nutrición y

Tecnología de Alimentos

(INTA) Universidad de

Chile.

aDoctora en Estadística.

${ }^{\text {b} M a t r o n a, ~ D o c t o r a ~ e n ~}$

Nutrición.

'Matrona, Magister en Salud Pública.

Financiado por el Fondo Nacional de Ciencia y Tecnología. Proyecto Fondecyt 1080589.

Recibido el 15 de marzo de 2011, aceptado el $1^{\circ}$ de agosto de 2011

Correspondencia a: Cecilia Albala E-mail: calbala@uchile.cl

\section{Socioeconomic inequalities in active life expectancy and disability related to obesity among older people}

Background: The rapid increase in life expectancy in Chile, with obesity as the main nutritional problem in all age groups, makes it necessary to ask whether the years gained are healthy. Aim: To study the trajectories of disability associated with obesity in Chilean elderly groups from different socio-economic and demographic backgrounds. Material and Methods: Longitudinal study of 3 cohorts of older adults from Santiago: the SABE cohort including 1235 people born before 1940; the Alexandros cohort including 950 people born between 1940 and 1948 from Primary Health Care centers and the ISAPRES cohort of 266 people from private health insurance registries (ISAPRES) born before 1947. An interview yielded socio demographic data and history of diseases. Anthropometric measurements and hand dynamometry were performed. Cognitive status was assessed by the mini mental state examination, depressive symptoms through the geriatric depression socore-5 and functional limitations through self-reporting of basic ( $A D L)$, instrumental (IADL) and advanced daily living (AADL) activities. Results: We report here baseline results from ISAPRES and SABE cohorts. Important social and gender differentials were observed. After adjustment by age and gender, a significant lower frequency of limitations in $A D L$ (odds ratio $(O R)=$ 0.17 ; 95\% confidence intervals (CI): 0.079-0.343), IADL (OR =0.27; 95\% CI: 0.1590.452), and AADL (OR=0.42; 95\%CI: 0.298-0.599) persisted in the ISAPRE cohort, compared to the SABE cohort. Obesity was associated with functional limitations only in AADL (OR = 1.65; 95\%CI: 1.18-2.31) and hand dynamometry was associated with lower functional limitation in $A D L, I A D L$ and AADL. Conclusions: This study demonstrates profound socio-economic and gender inequalities in older people, thus showing that the years of healthy life gained are not the same for the whole society.

(Rev Med Chile 2011; 139: 1276-1285).

Key words: Disability evaluation; Life expectancy.
$\mathrm{E}$ n las últimas décadas, los países latinoamericanos han experimentado un acelerado proceso de transición demográfica y epidemiológica ${ }^{1,2}$, lo que ha originado un rápido envejecimiento de la población y un aumento en la frecuencia de enfermedades crónicas y degenerativas. En el período 1950-2010 la población de adultos mayores ( $\geq 60$ años) en Chile ha aumentado en $85 \%$, llegando a $13 \%$ de la población total en $2010^{3,4}$. En Latinoamérica, Chile es el país que 
ha aumentado más rápidamente la expectativa de vida al nacer (EVN). Entre los períodos 1970-75 y 2005-2010 la EVN aumentó de 60,5 a 75,5 años en hombres y de 66,8 a 81,5 años en mujeres ${ }^{3-5}$.

El deterioro gradual de las condiciones de salud física y mental que acompañan el envejecimiento individual, con el consecuente aumento de problemas de salud crónicos, conducen -de no mediar intervenciones específicas- a limitación funcional y gradual pérdida de autonomía. Las limitaciones funcionales, definidas como la restricción de las capacidades físicas o mentales para efectuar las tareas requeridas para mantener una vida independiente ${ }^{6}$, identifican un subgrupo importante de adultos mayores (AM) vulnerables y son importantes predictores de discapacidad, morbilidad y mortalidad en el $\mathrm{AM}^{7}$.

El gran éxito obtenido por la ciencia para mejorar la sobrevida humana, no significa necesariamente que los años ganados sean en buena salud. A medida que disminuye la mortalidad y aumenta la esperanza de vida (EV), cabe preguntarse sobre la calidad de los años ganados ${ }^{8}$. Los estudios efectuados en Europa occidental y Norteamérica muestran que el aumento de EV no ha sido acompañado por años con discapacidad sino que por el contrario, esta ha disminuido? Por otra parte, en países en desarrollo, en que el proceso de envejecimiento ocurre en un contexto de importantes desigualdades, la mayor sobrevida se acompaña de mayor limitación funcional en los grupos socialmente vulnerables ${ }^{10}$. Las desigualdades socioeconómicas y de género observadas en los AM, hacen prever un aumento de la discapacidad y limitaciones funcionales en este grupo, ya que éstas surgen tanto de procesos patológicos, como también de conductas o factores sociales, dentro de los cuales la nutrición juega un importante papel.

La obesidad se ha ligado a expectativas de vida más largas ${ }^{11,12}$, sin embargo, la mayor parte de los estudios la asocian con limitación funcional, señalando que los IMC mayores se asocian con discapacidad, aunque los pesos menores también se han asociado a limitación funcional ${ }^{13-15}$.

La epidemia de obesidad que afecta a la población nacional y las conocidas asociaciones de la obesidad con riesgos para la salud, hacen temer que en las próximas generaciones de AM se observen serias consecuencias sobre la funcionalidad. En Chile, la obesidad, definida por IMC $\geq 30 \mathrm{~kg} / \mathrm{m}^{216}$, es muy prevalente en AM. La Encuesta Nacional de
Salud (ENS) $2009^{17}$, encontró una prevalencia de obesidad de $35,5 \%$ en mujeres y $24,6 \%$ en hombres mayores de 64 años.

Dados la alta prevalencia y los efectos negativos de la obesidad sobre la funcionalidad, en una creciente población mayor con profundas desigualdades socioeconómicas, se espera un aumento en las tasas de discapacidad, con la consiguiente pérdida de años saludables.

Nuestra hipótesis plantea que la ganancia de años de vida de los AM en Chile, no ha sido acompañada por una disminución de la discapacidad y que la ganancia de años saludables presenta una distribución desigual entre los AM de distintos estratos socioeconómicos. Proponemos que la obesidad es un factor de riesgo para limitación funcional y discapacidad y que la mayor expectativa de vida observada en los obesos, es debida al origen demográfico de las cohortes estudiadas.

\section{Población y Métodos}

El presente estudio se refiere al análisis basal de las cohortes SABE e ISAPRES, que forman parte del proyecto ALEXANDROS. Dicho proyecto corresponde a un estudio de cohorte prospectivo en el cual se comparan 3 cohortes de AM provenientes de diferentes contextos demográficos y socioeconómicos.

1. La cohorte del estudio $S A B E^{18}$, a la cual pertenecen AM nacidos antes de 1940, que incluye 1235 personas de 60 años y más, seleccionados en una muestra probabilística y representativa del Gran Santiago, evaluados en 2000 y 2004/2005.

2. La cohorte Alexandros está compuesta por 950 sujetos nacidos desde 1940 a 1948, seleccionados al azar entre los AM registrados en 28 centros de atención primaria del Gran Santiago. Este grupo permitirá estudiar el efecto del origen demográfico en comparación con la cohorte SABE. La cohorte se compone de:

- 491 sujetos nacidos entre 1940 y 1942, elegidos aleatoriamente de la cohorte CENEX $^{19}$, con datos basales de 2005/2006.

- 459 AM nacidos entre 1943 y 1948, reclutados entre sujetos registrados en los mismos 28 centros de salud seleccionados en el estudio CENEX y seleccionados de la misma forma que ellos, con evaluación basal en 2008 . 
3. La cohorte ISAPRES, constituida por 204 AM nacidos antes de 1948, seleccionados en forma aleatoria de la base de beneficiarios de ISAPRES abiertas del Gran Santiago ${ }^{20}$, que fueron evaluados entre 2006 y 2007 .

Esta cohorte permitirá determinar diferenciales socioeconómicos con la cohorte SABE.

\section{Protocolo de investigación}

Los participantes respondieron un cuestionario con datos socio-demográficos, historia de enfermedades, auto-reporte de limitaciones funcionales y los tests Mini Mental State Examination (MMSE) ${ }^{21-23}$, cuestionario de actividades funcionales de Pffefer ${ }^{22,24}$ y escala de depresión geriátrica abreviada (GDS-5) ${ }^{25,26}$. Un profesional entrenado y estandarizado para el estudio realizó evaluación antropométrica, dinamometría y medición de presión arterial. Todos los participantes firmaron un consentimiento después de recibir información verbal y escrita del estudio, cuyo protocolo fue aprobado por el Comité de Ética del INTA.

\section{Evaluación antropométrica}

La talla fue medida con el sujeto de pie y descalzo, utilizando un Estadiómetro Harpender (Holtain Ltd., Crosswell, G.B.). El peso fue medido con una balanza SECA (Madison, WI, U.S.A.) graduada a $0,1 \mathrm{~kg}$ con el sujeto de pie y descalzo. Las circunferencias de cintura y caderas se midieron con huincha ad-hoc SECA. La medición se efectuó, en un plano paralelo al piso, inmediatamente por encima de la cresta ilíaca para la circunferencia de cintura y en la parte más prominente de las nalgas, para la circunferencia de caderas. La fuerza muscular se evaluó con 2 mediciones de dinamometría (Hand Dinamometer T-18; Country Technology, Inc.) en la mano dominante, registrando la marca mayor. Se calculó el IMC para categorizar el estado nutricional de acuerdo a criterio OMS ${ }^{16}$.

\section{Limitaciones funcionales y capacidades físicas}

La evaluación de la funcionalidad se efectuó por autoreporte de limitaciones en: actividades básicas de la vida diaria (ABVD) como transferirse desde la silla a la cama y viceversa, comer, caminar dentro de una pieza, vestirse, bañarse y usar el excusado $^{27}$; limitaciones en actividades instrumentales de la vida diaria (AIVD) como realizar quehaceres livianos y pesados del hogar, lavar, cocinar, compra de almacén y alimentos, manejo de dinero, tomar sus medicamentos, llamar por teléfono y salir a lugares cercanos ${ }^{28}$ y limitaciones en movilidad o actividades avanzadas de la vida diaria (AAVD) como subir escalas, caminar, agacharse, levantar los brazos por sobre el nivel de la cabeza, levantar $5 \mathrm{~kg}$ de peso y recoger una moneda desde una superficie lisa ${ }^{29,30}$.

\section{Análisis estadístico}

En el presente estudio informamos sobre las diferencias basales entre las cohortes SABE e ISAPRES. Para el análisis presente, se incluyeron los 62 sujetos afiliados a ISAPRE de la cohorte SABE, en la cohorte ISAPRES. Para la descripción basal se calcularon las medias, desviación estándar (DS), rangos e intervalos de confianza (IC) al 95\% y distribuciones percentilares. Las variables estudiadas en ambas cohortes se compararon mediante $t$ test o estadístico $z$ según fueran continuas o categóricas. La asociación entre limitación funcional y estado nutricional se evaluó mediante test $\mathrm{chi}^{2}$. La asociación independiente de limitación funcional con cohorte y variables antropométricas ajustadas por edad y sexo, se efectuó por regresión logística. El diagnóstico de síntomas depresivos se efectuó con puntaje 2 o más en GDS- $5^{26}$ y el deterioro cognitivo con puntaje $<13$ en el MMSE abreviado en la cohorte SABE y $<15$ en la cohorte ISAPRES ${ }^{20,23}$.

Los análisis se realizaron con el programa Stata 10.1

\section{Resultados}

Las características sociodemográficas de las cohortes se describen en la Tabla 1. La cohorte ISAPRE es 2 años más joven $(p=0,01)$ y tiene una proporción de mujeres similar $(\mathrm{p}=0,095)$ a la cohorte SABE. Destacan las grandes diferencias en el nivel educacional entre ambos grupos, con un promedio de 5,3 años de escolaridad en la cohorte SABE y 13 en la cohorte ISAPRES. La proporción de sujetos que vive solo es similar, aunque la proporción de casados o en pareja es muy superior en ISAPRES y la proporción de viudos y separados es significativamente mayor en la cohorte SABE. En la Tabla 2 se describe la frecuencia de enfermedades crónicas auto-reportadas según sexo, en ambas cohortes. En la cohorte SABE las mujeres presentan mayor número de enfermedades que los hombres y la frecuencia de las enfermedades estudiadas es 
ARTÍCULOS DE INVESTIGACIÓN

Desigualdades socioeconómicas en expectativa de vida y discapacidad en adultos mayores - C. Albala et al

Tabla 1. Características sociodemográficas de los adultos mayores estudiados según cohorte de origen y sexo

\begin{tabular}{|c|c|c|c|c|c|c|}
\hline & \multicolumn{3}{|c|}{ SABE } & \multicolumn{3}{|c|}{ ISAPRES } \\
\hline & $\begin{array}{l}\text { Hombres } \\
\text { n: } 397\end{array}$ & $\begin{array}{l}\text { Mujeres } \\
n=776 \\
(66,2 \%)\end{array}$ & $\begin{array}{c}\text { Total } \\
\text { n }=1.173\end{array}$ & $\begin{array}{c}\text { Hombres } \\
n=105\end{array}$ & $\begin{array}{c}\text { Mujeres } \\
n=161 \\
(60,5 \%)\end{array}$ & $\begin{array}{c}\text { Total } \\
n=266\end{array}$ \\
\hline $\begin{array}{l}\text { Edad } \\
\text { promedio } \pm \text { DE }(95 \% \mid C)\end{array}$ & $\begin{array}{l}70,8 \pm 7,5^{a} \\
(70,0-71,5)\end{array}$ & $\begin{array}{l}72,2 \pm 8,3^{a, b} \\
(71,6-72,7)\end{array}$ & $\begin{array}{l}71,7 \pm 8,1^{d} \\
(71,3-72,2)\end{array}$ & $\begin{array}{l}69,6 \pm 7,2 \\
(68,2-71,0)\end{array}$ & $\begin{array}{l}69,6 \pm 6,5^{b} \\
(68,6-70,6)\end{array}$ & $\begin{array}{l}69,6 \pm 6,8^{d} \\
(68,8-70,5)\end{array}$ \\
\hline $\begin{array}{l}\text { No puede leer ni escribir } \\
\%(95 \% \mid C)\end{array}$ & $\begin{array}{c}10,0 \text { ac } \\
(7,2-13,5)\end{array}$ & $\begin{array}{c}18,0^{\mathrm{a}, \mathrm{b}} \\
(15,3-20,8)\end{array}$ & $\begin{array}{c}15,3^{d} \\
(13,3-17,5)\end{array}$ & $\begin{array}{c}0,95^{c} \\
(0,2-5,2)\end{array}$ & $\begin{array}{c}1,24^{b} \\
(0,01-4,4)\end{array}$ & $\begin{array}{c}1,1^{d} \\
(0,2-3,3)\end{array}$ \\
\hline $\begin{array}{l}\text { Educación } \\
\text { Años educación } \\
\text { promedio } \pm \text { DE }(95 \% \text { IC) }\end{array}$ & $\begin{array}{c}6,1 \pm 4,6 \text { ac } \\
(5,7-6,6)\end{array}$ & $\begin{array}{c}5,0 \pm 4,1^{a, b} \\
(4,7-5,3)\end{array}$ & $\begin{array}{c}5,3 \pm 4,3^{d} \\
(5,1-5,6)\end{array}$ & $\begin{array}{l}14,1 \pm 3,6 c \\
(13,4-14,9)\end{array}$ & $\begin{array}{l}13,6 \pm 4,3^{b} \\
(12,9-14,4)\end{array}$ & $\begin{array}{l}13,0 \pm 4,2^{d} \\
(12,2-13,7)\end{array}$ \\
\hline $\begin{array}{l}<6 \text { años } \\
\%(95 \% \mid C)\end{array}$ & $\begin{array}{c}45,7^{\text {ac }} \\
(40,5-51,0)\end{array}$ & $\begin{array}{c}54,4^{\mathrm{a}, \mathrm{b}} \\
(50,8-58,0)\end{array}$ & $\begin{array}{c}51,6 \\
(48,6-54,6)\end{array}$ & $\begin{array}{c}2,8^{c} \\
(0,5-8,1)\end{array}$ & $\begin{array}{c}5,6^{\mathrm{b}} \\
(2,6-10,3)\end{array}$ & $\begin{array}{c}4,5 \\
(2,3-7,8)\end{array}$ \\
\hline $\begin{array}{l}\text { 6-12 años } \\
\%(95 \% \mid C)\end{array}$ & $\begin{array}{c}44,4 \\
(39,2-49,6)\end{array}$ & $\begin{array}{c}40,9 \\
(37,4-44,5)\end{array}$ & $\begin{array}{c}42,0 \\
(39,1-45,0)\end{array}$ & $\begin{array}{c}41,9 \\
(32,3-51,9)\end{array}$ & $\begin{array}{c}50,9 \\
(42,9-58,9)\end{array}$ & $\begin{array}{c}47,5 \\
(41,4-53,7)\end{array}$ \\
\hline $\begin{array}{l}13 \text { y más } \\
\%(95 \% \mid C)\end{array}$ & $\begin{array}{c}9,9^{a, c} \\
(7,0-13,4)\end{array}$ & $\begin{array}{c}4,6^{a, b} \\
(3,2-6,4)\end{array}$ & $\begin{array}{c}6,3 \\
(5,0-7,9)\end{array}$ & $\begin{array}{c}54,3 \mathrm{c} \\
(44,3-64,0)\end{array}$ & $\begin{array}{c}43,5^{b} \\
(35,7-51,5)\end{array}$ & $\begin{array}{c}47,9 \\
(41,7-54,1)\end{array}$ \\
\hline $\begin{array}{l}\text { Arreglos familiares } \\
\text { Vive solo \% } \\
(95 \% \mathrm{IC})\end{array}$ & $\begin{array}{c}9,54^{\mathrm{a}} \\
(6,8-12,9)\end{array}$ & $\begin{array}{c}13,6^{a} \\
(11,3-16,2)\end{array}$ & $\begin{array}{c}12,3 \\
(10,4-14,3)\end{array}$ & $\begin{array}{c}6,7 \\
(2,7-13,2)\end{array}$ & $\begin{array}{c}14,3 \\
(9,3-20,7)\end{array}$ & $\begin{array}{c}11,3 \\
(7,7-15,7)\end{array}$ \\
\hline $\begin{array}{l}\text { Estado civil } \\
\text { Casado o en pareja \% } \\
(95 \% \mid \mathrm{IC})\end{array}$ & $\begin{array}{c}52,4^{\mathrm{a}, \mathrm{c}} \\
(47,3-57,5)\end{array}$ & $\begin{array}{c}24,5^{\mathrm{ab}} \\
(20,3-29,1)\end{array}$ & $\begin{array}{c}33,7^{d} \\
(31,0-36,5)\end{array}$ & $\begin{array}{c}70,9^{c} \\
(54,4-80,5)\end{array}$ & $\begin{array}{c}63, \mathrm{~b} \\
(49,1-74,9)\end{array}$ & $\begin{array}{c}66,7^{d} \\
(56,0-74,2)\end{array}$ \\
\hline $\begin{array}{l}\text { Soltero } \\
\%(95 \% \mid C)\end{array}$ & $\begin{array}{c}3,6^{\mathrm{a}, \mathrm{c}} \\
(2,0-6,0)\end{array}$ & $\begin{array}{c}9,3^{\mathrm{a}} \\
(7,4-11,6)\end{array}$ & $\begin{array}{c}7,4^{d} \\
(6,0-9,1)\end{array}$ & $\begin{array}{c}14,7^{c} \\
(2,0-17,9)\end{array}$ & $\begin{array}{c}12,2 \\
(10,6-14,1)\end{array}$ & $\begin{array}{c}13,4^{d} \\
(7,6-20,9)\end{array}$ \\
\hline $\begin{array}{l}\text { Separado } \\
\%(95 \% \mid C)\end{array}$ & $\begin{array}{c}19,4^{\mathrm{a}, \mathrm{c}} \\
(15,5-23,7)\end{array}$ & $\begin{array}{c}19,0^{\mathrm{b}} \\
(16,3-21,9)\end{array}$ & $\begin{array}{c}19,1^{d} \\
(16,9-21,5)\end{array}$ & $\begin{array}{c}8,5^{\mathrm{a}, \mathrm{c}} \\
(4,2-22,6)\end{array}$ & $\begin{array}{c}0,5^{\mathrm{a}, \mathrm{b}} \\
(0,04-9,1)\end{array}$ & $\begin{array}{c}4,2^{\mathrm{d}} \\
(2,5-12,3)\end{array}$ \\
\hline $\begin{array}{l}\text { Viudo } \\
\%(95 \% \mid \mathrm{l})\end{array}$ & $\begin{array}{c}24,5^{a, c} \\
(21,5-27,6)\end{array}$ & $\begin{array}{c}47,2^{\mathrm{a}, \mathrm{b}} \\
(43,6-50,7)\end{array}$ & $\begin{array}{c}39,7^{\mathrm{d}} \\
(36,-42,6)\end{array}$ & $\begin{array}{c}5,9^{\mathrm{a}, \mathrm{c}} \\
(5,4-24,9)\end{array}$ & $\begin{array}{c}24,2^{a, b} \\
(19,2-43,9)\end{array}$ & $\begin{array}{c}15,7^{d} \\
(15,4-25,2)\end{array}$ \\
\hline
\end{tabular}

${ }^{a}$ Diferencia entre hombres y mujeres intra cohorte $<0,05$; ${ }^{b}$ Diferencia mujeres intercohorte $<0,05$; ${ }^{\circ}$ Diferencia hombres in-

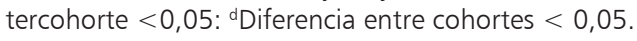

mayor o similar a la de los hombres. En la cohorte ISAPRE el número de enfermedades crónicas es similar en ambos sexos, pero los hombres superan a las mujeres en frecuencia de diabetes y las mujeres tienen mayor frecuencia de enfermedades osteoarticulares y síntomas depresivos. La frecuencia de caídas, la hipertensión y la enfermedad pulmonar obstructiva crónica (EPOC) son más frecuentes en mujeres de la cohorte SABE que en las de la cohorte ISAPRE.

En la Tabla 3 se describe la autoevaluación de la salud y la funcionalidad física y mental de los AM estudiados. En la cohorte SABE las mujeres presentan mayor frecuencia de alteración en todos los indicadores de limitación funcional descritos. En la cohorte ISAPRES, la funcionalidad es similar en hombres y mujeres. Cuando se comparan mujeres de ambas cohortes, se observa que las mujeres ISAPRE tienen indicadores mejores que las mujeres $\mathrm{SABE}$ en todos los ítems estudiados. Algo similar ocurre con los hombres de ambas cohortes, excepto en deterioro cognitivo.

En la Tabla 4 se describen las características antropométricas y de estado nutricional. Los hombres de ISAPRE tienen mayor peso, talla, IMC y dinamometría que los hombres de SABE. En el caso de las mujeres, aquellas de la cohorte $\mathrm{SABE}$ tienen mayor peso, IMC, circunferencia de cintura 
ARTÍCULOS DE INVESTIGACIÓN

Desigualdades socioeconómicas en expectativa de vida y discapacidad en adultos mayores - C. Albala et al

Tabla 2. Frecuencia de morbilidad de los adultos mayores estudiados según cohorte de origen y sexo

\begin{tabular}{|c|c|c|c|c|c|c|c|c|}
\hline \multirow{3}{*}{$\begin{array}{l}\text { Enfermedades } \\
\%(95 \% \mathrm{IC}) \\
\text { Hipertensión }\end{array}$} & \multicolumn{4}{|c|}{$\begin{array}{c}\text { SABE } \\
n=1.159\end{array}$} & \multicolumn{4}{|c|}{$\begin{array}{l}\text { ISAPRES } \\
n=266\end{array}$} \\
\hline & \multicolumn{2}{|c|}{$\begin{array}{c}\text { Hombres } \\
n=383\end{array}$} & \multicolumn{2}{|c|}{$\begin{array}{l}\text { Mujeres } \\
\mathrm{n}=776\end{array}$} & \multicolumn{2}{|c|}{$\begin{array}{c}\text { Hombres } \\
n=105\end{array}$} & \multicolumn{2}{|c|}{$\begin{array}{l}\text { Mujeres } \\
n=161\end{array}$} \\
\hline & $70,4^{a, c}$ & $(65,5-74,9)$ & $76,6^{a, b}$ & $(73,4-79,4)$ & $36,2^{c}$ & $(27,0-46,1)$ & $39,1^{b}$ & $(31,5-47,1)$ \\
\hline Diabetes & $11,7^{c}$ & $(8,7-15,4)$ & 14,0 & $(11,7-16,7)$ & $21,9^{a, c}$ & $(13,6-30)$ & $11,2^{\mathrm{a}}$ & $(6,8-17,1)$ \\
\hline Síntomas depresivos & $37,6^{c}$ & $(34,0-41,3)$ & 32,3 & $(27,5-37,4)$ & $13,6^{a, c}$ & $(7,6-21,7)$ & $26,3^{a}$ & $(29,4-48,9)$ \\
\hline Osteoartritis & $13,7^{\mathrm{a}}$ & $(10,4-17,5)$ & $40,9^{a}$ & $(37,4-44,4)$ & $20,0^{a}$ & $(12,8-28,9)$ & $44,7^{a}$ & $(36,9-52,7)$ \\
\hline Cáncer & $3,4^{c}$ & $(1,8-5,7)$ & 5,4 & $(3,9-7,2)$ & $11,4^{c}$ & $(6,0-19,1)$ & 9,3 & $(5,3-14,9)$ \\
\hline EPOC & $13,9 c$ & $(10,6-17,8)$ & $12,7^{b}$ & $(10,5-15,3)$ & $2,9^{c}$ & $(0,6-8,1)$ & $5,6^{b}$ & $(2,6-10,4)$ \\
\hline Enfermedad coronaria & $29,6^{\text {ac }}$ & $(25,1-34,4)$ & $34,3^{a, b}$ & $(30,9-37,7)$ & $10,5^{c}$ & $(5,4-18,0)$ & $12,4^{b}$ & $(7,8-18,5)$ \\
\hline Accidente vascular encefálico & 6,0 & $(3,8-8,8)$ & 6,8 & $(5,1-8,7)$ & 2,9 & $(0,6-8,1)$ & 2,5 & $(0,7-6,2)$ \\
\hline Caídas últimos 6 meses & $28,1^{\text {a }}$ & $(23,7-32,8)$ & $40,2^{a, b}$ & $(36,7-43,7)$ & 19,1 & $(12,0-27,9)$ & $29,2^{b}$ & $(22,3-36,9)$ \\
\hline $\begin{array}{l}\text { Enfermedades crónicas } \\
\text { Ninguna }\end{array}$ & $15,2^{a, c}$ & $(11,8-19,2)$ & $8,3^{a, b}$ & $(6,4-10,4)$ & $26,7^{c}$ & $(18,5-36,2)$ & $18,6^{b}$ & $(12,9-25,5)$ \\
\hline Una & $43,0^{a}$ & $(38,0-48,1)$ & $33,2^{\mathrm{a}}$ & $(29,9-36,7)$ & 41,9 & $(32,3-51,9)$ & 37,9 & $(30,4-45,8)$ \\
\hline 2 y más & $41,7^{a}$ & $(36,8-46,8)$ & $58,5^{a, b}$ & $(54,9-61,9)$ & 31,4 & $(22,7-41,2)$ & $43,5^{b}$ & $(35,7-51,5)$ \\
\hline
\end{tabular}

a Diferencia entre hombres y mujeres intra cohorte $<0,05$; ${ }^{b}$ Diferencia entre mujeres intercohorte $<0,05$; ${ }^{c}$ Diferencia entre hombres intercohorte $<0,05$.

Tabla 3. Estado funcional de los adultos mayores estudiados según cohorte de origen y sexo

\begin{tabular}{|c|c|c|c|c|c|c|c|c|}
\hline & \multicolumn{4}{|c|}{ SABE } & \multicolumn{4}{|c|}{ ISAPRES } \\
\hline & \multicolumn{2}{|c|}{$\begin{array}{c}\text { Hombres } \\
n=388\end{array}$} & \multicolumn{2}{|c|}{$\begin{array}{l}\text { Mujeres } \\
n=784\end{array}$} & \multicolumn{2}{|c|}{$\begin{array}{l}\text { Hombres } \\
n=105\end{array}$} & \multicolumn{2}{|c|}{$\begin{array}{l}\text { Mujeres } \\
n=161\end{array}$} \\
\hline Buena \% $(95 \% \mid C)$ & $40,7^{c}$ & $(35,8-45,8)$ & $31,1^{a, b}$ & $(27,9-34,5)$ & $77,1^{\mathrm{c}}$ & $(67,9-84,8)$ & $74,5^{b}$ & $(67,1-81,1)$ \\
\hline Regular \% (95\%|C) & $40,2^{c}$ & $(35,3-45,3)$ & $45,1^{\mathrm{b}}$ & $(41,6-48,7)$ & $20,0^{c}$ & $(12,8-28,9$ & $19,9^{b}$ & $(14,0-26,9)$ \\
\hline Mala \% (95\%lC) & $19,1^{c}$ & $(15,3-23,3)$ & $23,7^{b}$ & $(20,8-26,9)$ & $2,8^{c}$ & $(0,5-8,1)$ & $5,6^{b}$ & $(2,6-10,3)$ \\
\hline Deterioro cognitivo \% (95\%।C) & $8,5^{a}$ & $(5,9-11,7)$ & $14,7^{a, b}$ & $(12,3-17,3)$ & 3,8 & $(1,0-9,5)$ & $3,7^{b}$ & $(1,4-7,9)$ \\
\hline $\begin{array}{l}\text { Limitación en } \\
1 \text { o más ABVD \% (95\%IC) }\end{array}$ & $15,2^{\mathrm{a}, \mathrm{c}}$ & $(11,8-19,2)$ & $27,8^{a, b}$ & $(24,7-31,0)$ & $3,8^{c}$ & $(1,0-9,5)$ & $2,5^{b}$ & $(0,7-6,2)$ \\
\hline $\begin{array}{l}\text { Limitación funcional } \\
1 \text { o más AIVD \% (95\%|C) }\end{array}$ & $18,1^{\mathrm{a}, \mathrm{c}}$ & $(14,4-22,3)$ & $35,6^{a, b}$ & $(32,3-39,1)$ & $5,7^{c}$ & $(2,1-12,2)$ & $7,6^{b}$ & $(4,0-13,0)$ \\
\hline $\begin{array}{l}\text { Limitación funcional } \\
1 \text { o más AAVD \% }(95 \% \mid C)\end{array}$ & $54,7^{\mathrm{a}, \mathrm{c}}$ & $(49,4-59,9)$ & $77,0^{\mathrm{a}, \mathrm{b}}$ & $(73,7-80,1)$ & $10,6^{c}$ & $(5,4-18,1)$ & $19,7^{b}$ & $(13,7-27,0)$ \\
\hline
\end{tabular}

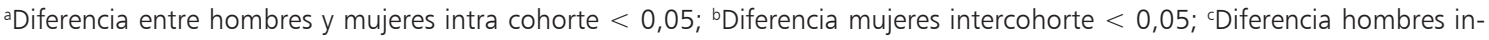
tercohorte $<0,05$. 
ARTÍCULOS DE INVESTIGACIÓN

Desigualdades socioeconómicas en expectativa de vida y discapacidad en adultos mayores - C. Albala et al

Tabla 4. Estado nutricional y variables antropométricas de los adultos mayores estudiados según cohorte y sexo

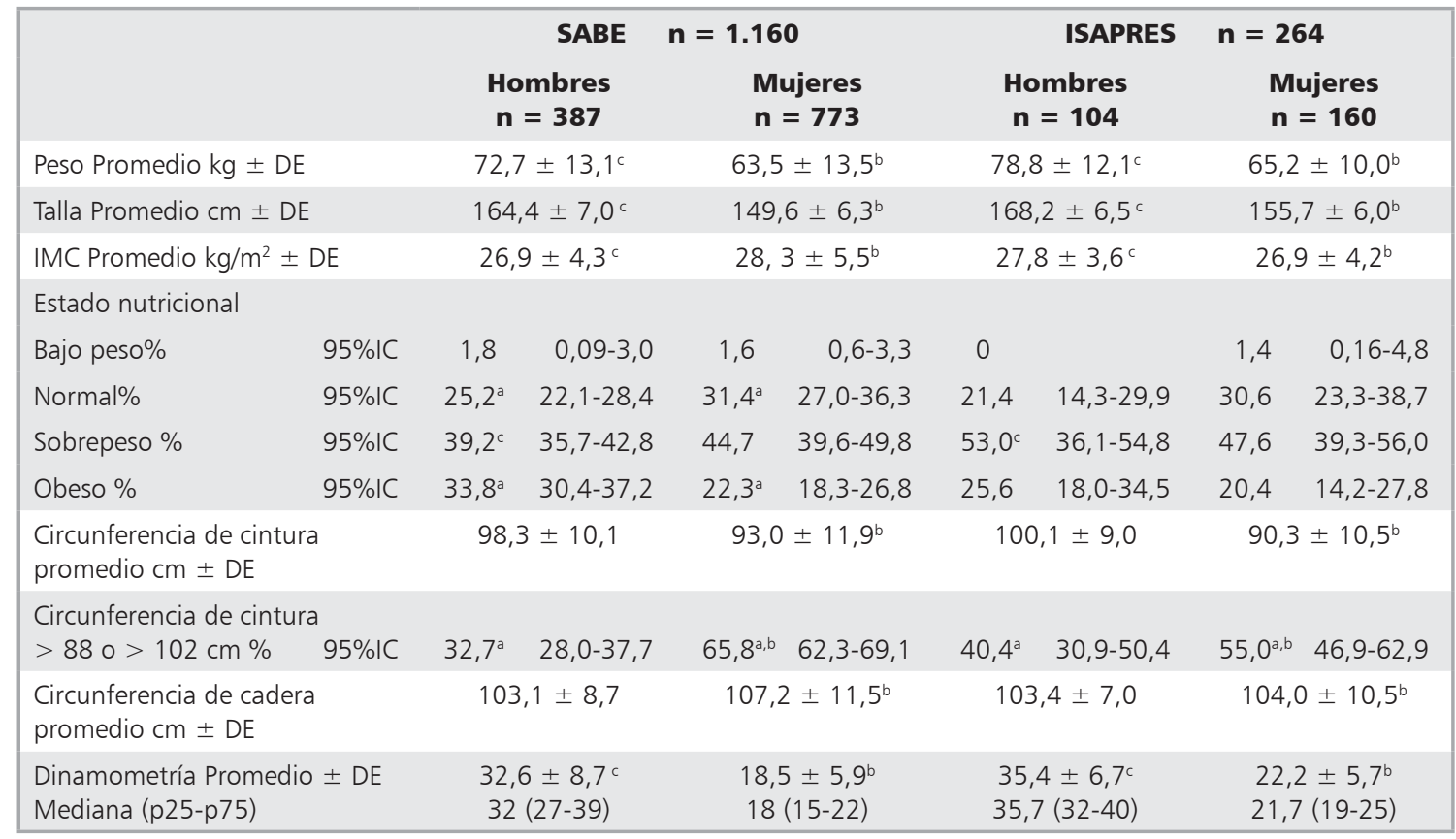

Diferencia proporciones estadístico z. Diferencia de promedios, estadístico t; a Diferencia entre hombres y mujeres intra cohorte $p<0,05$; ${ }^{b}$ Diferencia mujeres intercohorte $<0,05$; ${ }^{~}$ Diferencia hombres intercohorte $<0,05$.

Tabla 5. Frecuencia de limitación funcional según estado nutricional* de los adultos mayores según sexo y cohorte

\begin{tabular}{|c|c|c|c|c|c|c|}
\hline \multirow[b]{2}{*}{ IMC kg/m² } & \multicolumn{3}{|c|}{ SABE } & \multicolumn{3}{|c|}{ ISAPRES } \\
\hline & 1ABVD & 1 AIVD & 2 AAVD & 1ABVD & 1 AIVD & 2 AAVD \\
\hline Hombres & $\%(95 \% \mid C)$ & $\%(95 \% \mid C)$ & $\%(95 \% \mid C)$ & $\%(95 \% \mid C)$ & $\%(95 \% \mid C)$ & $\%(95 \% \mid C)$ \\
\hline $18,5-24,9$ & $\begin{array}{c}14,9 \\
(9,1-20,3)\end{array}$ & $\begin{array}{c}26,7 \\
(19,0-35,5)\end{array}$ & $\begin{array}{c}32,4 \\
(23,7-42,1)\end{array}$ & $\begin{array}{c}5,3 \\
(0,1-26,0)\end{array}$ & $\begin{array}{c}5,3 \\
(0,1-26,0)\end{array}$ & $\begin{array}{c}21,0 \\
(6,0-45,6)\end{array}$ \\
\hline $25-29,9$ & $\begin{array}{c}15,7 \\
(10,6-22,0)\end{array}$ & $\begin{array}{c}14,6 \\
(9,7-20,8)\end{array}$ & $\begin{array}{c}27,5 \\
(20,7-35,1)\end{array}$ & $\begin{array}{c}5,1 \\
(1,1-14,2)\end{array}$ & $\begin{array}{c}8,5 \\
(2,8-18,7)\end{array}$ & $\begin{array}{c}10,3 \\
(3,9-21,2)\end{array}$ \\
\hline$\geq 30$ & $\begin{array}{c}11,6 \\
(5,7-20,3)\end{array}$ & $\begin{array}{c}10,5 \\
(4,9-18,9)\end{array}$ & $\begin{array}{c}21,4 \\
(13,2-31,7)\end{array}$ & 0 & 0 & $\begin{array}{c}11,5 \\
(2,4-30,1)\end{array}$ \\
\hline Test exacto de Fisher & 0,692 & 0,005 & 0,244 & 0,632 & 0,441 & 0,469 \\
\hline \multicolumn{7}{|l|}{ Mujeres } \\
\hline $18,5-24,9$ & $\begin{array}{c}29,4 \\
(23,1-36,3)\end{array}$ & $\begin{array}{c}40,9 \\
(33,9-48,2)\end{array}$ & $\begin{array}{c}53,0 \\
(45,1-60,7)\end{array}$ & $\begin{array}{c}3,9 \\
(0,5-13,5)\end{array}$ & $\begin{array}{c}8,0 \\
(2,2-19,2)\end{array}$ & $\begin{array}{c}18,4 \\
(8,7-32,0)\end{array}$ \\
\hline $25-29,9$ & $\begin{array}{c}23,5 \\
(18,8-28,7)\end{array}$ & $\begin{array}{c}29,2 \\
(24,1-34,7)\end{array}$ & $\begin{array}{c}49,2 \\
(43,1-55,4)\end{array}$ & $\begin{array}{c}2,7 \\
(0,3-9,6)\end{array}$ & $\begin{array}{c}9,9 \\
(4,0-19,2)\end{array}$ & $\begin{array}{c}24,3 \\
(14,8-36,0)\end{array}$ \\
\hline$\geq 30$ & $\begin{array}{c}26,5 \\
(21,3-32,4)\end{array}$ & $\begin{array}{c}35,8 \\
(29,9-41,9)\end{array}$ & $\begin{array}{c}59,2 \\
(52,6-65,4)\end{array}$ & 0 & $\begin{array}{c}2,9 \\
(0,07-15,3)\end{array}$ & $\begin{array}{c}39,4 \\
(22,9-57,9)\end{array}$ \\
\hline Test exacto de Fisher & 0,334 & 0,024 & 0,080 & 0,818 & 0,586 & 0,105 \\
\hline
\end{tabular}

*No se incluyeron los sujetos con IMC $<18,5$ porque su bajo número ( 2 en Isapres y 19 en SABE), no permite hacer comparaciones. 
Tabla 6. Análisis de regresión logística para diferentes categorías de limitación funcional como variables dependientes y variables antropométricas y cohorte de origen como variables independientes, ajustado por sexo y edad

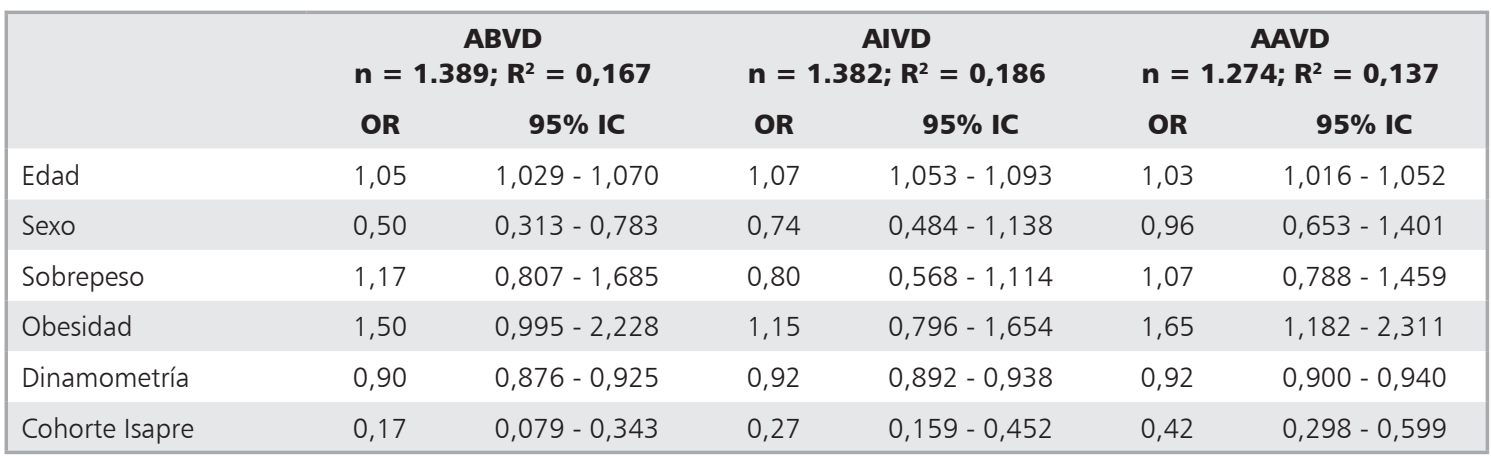

Test Bondad de Ajuste Hosmer-Lemeshow $\chi^{2},>0,24$ para las 4 regresiones.

y de caderas que las de ISAPRE, pero menor talla y menor dinamometría que las últimas. El estado nutricional de las mujeres es similar en ambas cohortes, pero los hombres de ISAPRE tienen mayor proporción de sobrepeso que los hombres de la cohorte SABE.

Las asociaciones crudas entre categorías de IMC y limitación funcional en ambas cohortes muestran asociación significativa sólo para limitación en 1 o más AIVD en hombres y mujeres de la cohorte SABE (Tabla 5).

Para estudiar la asociación independiente de los diferentes factores estudiados con limitación funcional, se efectuaron análisis de regresión múltiple para limitación en 1 o más AVD, 1 o más AIVD y 2 o más AAVD incluyendo: edad, sexo, estado nutricional, dinamometría y cohorte. Para todas las limitaciones estudiadas, la pertenencia a la cohorte ISAPRE y una mayor dinamometría son factores protectores. La edad se asocia positivamente con todas las limitaciones funcionales, no así género femenino. La obesidad se asoció sólo con limitación en AAVD (Tabla 6).

\section{Discusión}

Los resultados del presente estudio revelan que la frecuencia de limitaciones funcionales es muy superior en la cohorte SABE que en la cohorte ISAPRE, con diferencias de género sólo en la cohorte SABE. Ello concuerda con las diferencias abismantes en nivel educacional entre ambas co- hortes, pero también a que en la cohorte ISAPRE no hubo diferencias de educación entre hombres y mujeres. Diversos estudios han demostrado que las limitaciones funcionales surgen no sólo como consecuencia de procesos patológicos, sino también como resultado de conductas o factores sociales $^{31}$, habiéndose descrito tasas mayores en los más viejos, en las minorías y en los más pobres $^{6,32}$. En estudios previos sobre mortalidad en el gran Santiago, hemos encontrado que en los AM no hay diferencias en la mortalidad de los distintos estratos socio-económicos, observándose una equidad casi perfecta para los mayores de 80 años $^{33}$, sin embargo, en lo referente a limitaciones funcionales hay grandes desigualdades. En el estudio SABE se observó una prevalencia significativamente mayor de discapacidad en los AM de mayor edad, en los con menor escolaridad y en las mujeres, ${ }^{9,34}$. Hallazgos similares describe Kuh en una muestra del Reino Unido, donde las mujeres tenían serias desventajas en relación a los hombres en funcionalidad física, especialmente en los más pobres, en obesos y en sedentarios ${ }^{35}$. Ello avala la importancia de aspectos socioeconómicos y el papel de la nutrición y la actividad física en el envejecimiento saludable.

La comparación de indicadores antropométricos y funcionales entre ambas cohortes muestra una estrecha asociación de la funcionalidad con el nivel socioeconómico, representado por la adscripción al sistema público o privado de salud. Una debilidad de este reporte es su carácter transversal, pero se trata de una comparación basal, en un es- 
tudio en que esperamos aportar mayor evidencia a través de la observación longitudinal, lo que nos permitirá evaluar la transición entre los estados de autonomía y discapacidad en las diferentes cohortes. Otra desventaja es el carácter selectivo de la muestra ISAPRE en la cual los sujetos con mayor carga de enfermedad se trasladan al subsector FONASA, al no poder costear las alzas de los planes. Este hecho radicaliza la situación de desigualdad económica entre ambas cohortes, extremando las diferencias socioeconómicas entre ambos grupos y dejando en el grupo ISAPRES los AM con menor carga de enfermedad. Considerando que en personas de 60 años y más la proporción de cotizantes de ISAPRE es $10,4 \%{ }^{36}$, una fortaleza del presente estudio es el importante número de personas reclutadas en esa cohorte. En los modelos de regresión múltiple, la edad, la dinamometría y la cohorte de origen mantuvieron una asociación independiente con funcionalidad para todos los indicadores de funcionalidad estudiados, encontrándose asociación con obesidad sólo para actividades más complejas como son las AAVD. Estos resultados concuerdan en parte con hallazgos previos que demuestran asociacion de obesidad con actividades de movilidad, con limitación funcional ${ }^{15,37} \mathrm{y}$ con discapacidad ${ }^{38}$. La fuerte asociación inversa entre dinamometría y funcionalidad ${ }^{34}$, demuestra que tanto la masa como la calidad y la fuerza muscular son factores determinantes de una funcionalidad adecuada. Estudios transversales han mostrado que la disminución de la masa muscular se asocia con un pobre rendimiento funcional y con autoreporte de discapacidad ${ }^{39,40}$ y que el músculo cambia produciéndose una creciente infiltración de grasa ${ }^{41,42}$. La epidemia de obesidad que afecta a todos los grupos etáreos y las conocidas asociaciones de la obesidad con riesgos para la salud, hacen temer que en las próximas generaciones de AM, con estilos de vida que han cambiado dramáticamente hacia conductas poco saludables ${ }^{2,43,44}$-de no mediar intervenciones-, se observen serias consecuencias sobre la funcionalidad. Existe evidencia que los programas destinados a aumentar la actividad física son efectivos en mejorar la movilidad y mantienen la autonomía ${ }^{44}$. Por otra parte, el enfrentamiento de la obesidad en los AM es un tema complejo que debe ser abordado considerando los múltiples desafíos que representa la nutrición en el proceso de envejecimiento ${ }^{45}$.
La gran diferencia entre la prevalencia de limitación funcional en los afiliados al sistema público y aquellos afiliados al sistema privado de salud, en desmedro de las personas mayores adscritas al sistema público de salud, demuestra que los años de vida en buena salud ganados no son iguales para toda la sociedad y que de no existir intervenciones efectivas, el sistema público deberá enfrentarse a una escalada en la demanda de servicios y costos de salud.

\section{Referencias}

1. Albala C, Vio F. Epidemiologic Transition in Latin America: The Case of Chile. Public Health 1995; 109: 431-42.

2. Albala C, Vio F, Kain J, Uauy R. Nutrition Transition in Latin America: The Case of Chile. Nutr Reviews 2001; 56 (6): 170-6.

3. Instituto Nacional de Estadísticas INE Chile. Estimaciones y proyecciones de población por sexo y edad. Total país 1950-2050. INE F/CHI 1.92. INE 1995.

4. Instituto Nacional de Estadísticas INE Chile. Compendio estadístico 2010. INE 2010.

5. PAHO/WHO. Health Situation of the Americas. Basic Indicators 2006. PAHO/HDM/HA/06.01. Disponible en http://www.paho.org . Acceso enero 2010.

6. Pope AM, Tarlov AR. (1991). Disability in America: Toward a National Agenda for Prevention. Washington, DC: National Academy Press.

7. Verbrugge LM, Jette AM. The Disablement Process. Soc Sci Med 1994; 38: 1-14.

8. Robine JM, Romieu I, Cambois E. Les indicateurs d'esperance de sante Bulletin de l'Organisation mondiale de la Sante 1999. Recueil d'articles No 1: 107-11. En ingles en Bulletin of the World Health Organization 1999; 77: 181-5.

9. Christensen K, Doblhammer G, Rau R, Vaupel JW. Ageing populations: the challenges ahead. Lancet 2009; 374: 1196-208.

10. Albala C, García C, Lera L. Encuesta sobre salud, bienestar y envejecimiento en Santiago de Chile. Estudio SABE Chile. Instituto de Nutrición y Tecnología de los Alimentos Universidad de Chile. ISBN 978-956-19-0565-8. Santiago, Chile 2007.

11. Price GM, Uauy R, Breeze E, Bulpitt CJ, Fletcher AE. Weight, shape, and mortality risk in older persons: elevated waist hip ratio, not high body mass index, is associated with a greater risk of death. Am J Clin Nutr 2006; 84: 449-60.

12. Al Snih S, Ottenbacher KJ, Markides KS, Kuo Y-F, Es- 
chbach K, Goodwin JS. The effect of obesity on disability vs mortality in older Americans. Arch Intern Med 2007; 167: 774-80.

13. Roubenoff R. Sarcopenia: Effects on Body Composition and Function. J.Gerontol. A Biol Sci Med Sci 2003; 58: 1012-7.

14. Davidson KK, Ford ES, Cogswell ME, Dietz WH. Percentage of Body Fat and Body Mass Index are Associated with Mobility Limitations in People Aged 70 and Older from NHANES III. J Am Geriatr Soc 2002; 50: 1802-9.

15. Visser M, Langlois J, Guralnik JM Cauley JA, Kronmal RA, Robbins J et al. High Body Fatness but not Low Fat Free Mass Predicts Disability in Older Men and Women: the Cardiovascular Health Study. Am J Clin Nutr 1998; 68: 584-90.

16. WHO. World Health Organization. Obesity. Preventing and managing the global epidemic. Report of a WHO consultation on obesity. Geneva. 3-5 June, 1997.

17. Ministerio de Salud Chile. Resultados de la Encuesta nacional de enfermedades crónicas 2009. MINSAL 2009. Disponible en: www.minsal.cl/epidemiologia.

18. CENEX Study. Cost-effectiveness evaluation of a nutrition supplement and an exercise programme for older people in Chile: the CENEX study [ISRCTN48153354] 2007.

19. Evaluación Funcional del Adulto Mayor en Isapres, propuesta No 601-32-LE07, www.superdesalud.cl

20. Folstein MF, Folstein SE, McHugh PR.Mini-mental state. A practical method for grading the cognitive state of patients for the clinician. J Psychiatr Res 1975; 12 (3): 189-98.

21. Quiroga P, Albala C, Klaasen G. [Validation of a screening test for age associated cognitive impairment, in Chile]. Rev Med Chile 2004; 132: 467-78.

22. Albala C, Icaza G, Vio F, García C, Marín PP, Quiroga P, et al. A short test to evaluate cognitive impairment based on Folstein's MMSE. Gerontology 2001; 47 (S1): S183.

23. Pfeffer RI, Kurosaki TT, Harrah CH, Chance JM, Filos S. Measurement of functional Activities in older adults in the community. J Gerontol 1982; 37: 323-9.

24. Sheikh JI, Yesavage JA. Geriatric Depression Scale (GDS): Recent evidence and development of a shorter version. Clin Gerontol 1986; 5: 165-72.

25. Hoyl T, Valenzuela E, Marín PP. [Depression in the aged: preliminary evaluation of the effectiveness, as an screening instrument, of the 5-item version of the Geriatric Depression Scale]. Rev Med Chile 2000; 128: 1199-204.

26. Katz S, Akpom CA. A measure of primary sociobiological functions. Int J Health Serv 1976; 6: 493-509.

27. Lawton MP, Brody EM. Assessment of older people: Self maintaining and instrumental activities of daily living.
Gerontologist 1969; 9: 179-86.

28. Nagi SZ. Disability concepts revisited: implications for prevention. In Pope, A.M., \& Tarlov, A. R. eds (1991). Disability in America: Toward a National Agenda for Prevention. Washington, DC: National Academy Press.

29. Rosow I, Breslau MA.Guttman Health Scale for the Aged. J Gerontol 1966; 21: 556-9.

30. Lawrence RH, Jette AM. Disentangling the Disablement Process. J. Gerontol. B Psychol Sci Soc Sci 1996; 51: S173-82.

31. Tucker KL, Falcon LM, Bianchi LA, Cacho E \& Bermudez O. Self Reported Prevalence and Health Correlates of Functional Limitation among Massachusetts Elderly Puerto Ricans, Dominicans and a non-Hispanic White. J Gerontol A Biol Sci Med Sci 2000; 55 (2): M90-7.

32. Sánchez H, Albala C. Desigualdades en salud: mortalidad del adulto en comunas del Gran Santiago. Rev Med Chile 2004; 4: 453-60.

33. Arroyo P, Lera L, Sánchez H, Bunout D, Santos J, Albala C. Indicadores antropométricos, composición corporal y limitaciones funcionales en ancianos. Rev Med Chile 2007; 135: 846-54.

34. Kuh D, Bassey EJ, Butterworth S, Hardy R, Wadsworth M \& The Musculoskeletal Study Team. Grip Strength, Postural Control, and Functional Leg Power in a Representative Cohort of British Men and Women: Associations with Physical Activity, Health Status and Socioeconomic Conditions. J Gerontol A Biol Sci Med Sci 2005; 60: 22431.

35. Superintendencia de Salud. Boletín estadístico año 2009 de Isapres. Disponible en http://www.supersalud.cl/ documentacion/569/w3-article-6535.html [consultado el 12 marzo 2011].

36. Zoico E, Di Francesco V, Guralnik JM, Mazzali G, Bortolani A, Guariento S, et al. Physical Disability and Muscular Strength in Relation to Obesity and Different Body Composition Indexes in a Sample of Healthy Elderly Women. Int J Obes 2004; 28: 234-41.

37. Ferraro KF, Su YP, Gretebeck RJ, Black DR, Badylak SF. Body Mass Index and disability in adulthood: a 20-year panel study. Am J Public Health 2002; 92: 834-40.

38. Sternfeld B, Ngo L, Satariano WA, Tager IB. Associations of Body Composition with Physical Performance and Self-Reported Functional Limitation in Elderly Men and Women. Am J Epidemiol 2002; 156: 110-21.

39. Visser M, Kritchevsky SB, Goodpaster BH, Newman AB, Nevitt M, Stamm E, et al. Leg Muscle Mass and Composition in Relation to Lower Extremity Performance in Men and Women aged 70 to 79: the Health, Aging and Body Composition Study. J Am Geriatr Soc 2002; 50: 897-904. 
40. Forsberg AM, Nilsson E, Werneman J, Bergstrom J, Hultman E. Muscle Composition in Relation to Age and Sex. Clin Science 1991; 81: 249-56.

41. Overend TJ, Cunningham DA, Paterson DH, Lefcoe MS. Thigh Composition in Young and Elderly Men Determined by Computed Tomography. Clin Physiol 1992; 12: 629-40.

42. Mor V. The compression of morbidity hypothesis: a review of research and prospects for the future. J Am Geriatr Soc 2005; S308-9.

43. Vio F, Albala C, Kain J. Nutrition Transition in Chile
Revisited: Mid-Term Evaluation of Obesity Goals for the Period 2000-2010. Public Health Nutr 2008; 11: 405-12.

44. Dangour AD, Albala C, Allen E, Grundy E, Walker DG, Aedo C, et al. Effect of a nutrition supplement and physicalactivity program on pneumonia and walking capacity in chilean older people: afactorial cluster randomized trial. PLoS Med 2011; 8 (4): e1001023.

45. Johnson MA, Dwyer JT, Jensen GL, Miller JW, Speakman JR, Starke-Reed P, Volpi E. Challenges and new opportunities for clinical nutrition interventions in the aged. J Nutr 2011; 141: 535-41. 